\title{
Extracting Electron Densities in N-Type GaAs From Raman Spectra: Theory
}

\section{Herbert S. Bennett}

National Institute of Standards and Technology, Gaithersburg, MD 20899-8120

herbert.bennett@nist.gov
In this paper, we present the theory for calculating Raman line shapes as functions of the Fermi energy and finite temperatures in zinc blende, n-type GaAs for donor densities between $10^{16} \mathrm{~cm}^{-3}$ and $10^{19} \mathrm{~cm}^{-3}$. Compared to other theories, this theory is unique in two respects: 1) the many-body effects are treated self-consistently and 2) the theory is valid at room temperature for arbitrary values of the ratio $R=\left(Q^{2} / \alpha\right)$, where $Q$ is the magnitude of the normalized wave vector and $\alpha$ is the normalized frequency used in the Raman measurements. These calculations solve the charge neutrality equation self-consistently for a two-band model of GaAs at $300 \mathrm{~K}$ that includes the effects of high carrier concentrations and dopant densities on the perturbed densities of states used to calculate the Fermi energy as a function of temperature. The results are then applied to obtain the carrier concentrations from Fermi energies in the context of line shapes in Raman spectra due to the coupling between longitudinal optical phonons and plasmons. Raman measurements have been proposed as a non-destructive method for wafer acceptance tests of carrier density in semiconductor epilayers. The interpretation of Raman spectra to determine the majority electron density in $\mathrm{n}$ type semiconductors requires an interdisciplinary effort involving experiments, theory, and computer-based simulations and visualizations of the theoretical calculations.

Key words: complex dielectric response function; compound semiconductors; electron densities; line shape; non-destructive and contactless measurements; Raman spectra.

Accepted: August 17, 2007

Available online: http://www.nist.gov/jres

\section{Introduction}

The carrier concentration is a key figure of merit associated with a go/no-go decision for determining whether a wafer or an epitaxial layer meets specifications and should undergo further processing. Technology roadmaps from the microelectronics and nanomaterials industries [1-3] call for non-destructive and fast-turn around methods to measure transport properties such as carrier concentrations in semiconductor wafers and epitaxial layers. Non-destructive measurements are economically more significant for III-V compound semiconductor wafers with epitaxial layers than for Si-based wafers with epitaxial layers because the regions of wafers used for making contacts cannot be used for product. Contacting measurement methods may be acceptable for Si wafers, but such destructive methods are much less acceptable for III-V compound semiconductor wafers with epitaxial layers because Si wafers are much less expensive per unit area than compound semiconductor wafers.

Raman spectroscopy has been proposed as one possible way to measure carrier concentrations nondestructively. The shape of the Raman spectral lines due to the longitudinal-optical phonons interacting with the plasmon collective modes of the electron gas (so 
called coupled longitudinal optical (LO) phonon-plasmon modes) provides information on transport properties of the electron gas in polar semiconductors [4-8]. The frequencies $\omega$ of the coupled modes are proportional to carrier concentrations, and the peak widths $\Delta \omega$ of the coupled modes are proportional to the scattering rates due to electron-phonon interactions. The qualitative determination of carrier concentration and mobility, which is inversely proportional to the scattering rate, from Raman spectra is reasonably straightforward based on these proportionalities. The quantitative determination of carrier concentrations and mobilities requires more sophisticated modeling of the spectra. Many of these higher level models involve fitting the spectra with the Fermi energy as a parameter and then determining the carrier concentration from knowing the fitted Fermi energy [6].

Most interpretations of Raman measurements on compound semiconductors such as GaAs require physical models and associated input parameters that describe how carrier densities vary with dopant concentrations and Fermi energies. In this paper, we develop the theory for extracting electron densities from Raman measurements of n-type GaAs at room temperature. We introduce two main classes of models that relate carrier concentrations to the Fermi energy for a given temperature and donor dopant density:

a) Bandgap narrowing (BGN) models based on two equivalent bands at the $\Gamma$ point in the first Brilluoin zone, and

b) Parabolic densities of states (PDOS) models with and without a quartic term in the electron energy dispersion $E_{\mathrm{c \Gamma}}(\boldsymbol{k})$ for the $\Gamma$ conduction sub-band, where $\boldsymbol{k}$ is the wave vector.

The BGN models include many-body quantum effects and bandgap narrowing. The many-body quantum effects describe the electron-electron, electronhole, and dopant ion-carrier interactions. The results are unique in two respects: 1) the many-body effects are treated self-consistently and 2) the theory is valid at room temperature for arbitrary values of the ratio $R=$ $\left(Q^{2} / \alpha\right)$, where $Q$ is the magnitude of the normalized wave vector and $\alpha$ is the normalized frequency used in the Raman measurements. Other reported work either is valid at low temperatures near $0 \mathrm{~K}$ for arbitrary ratios $\left(Q^{2} / \alpha\right)[4]$ or is valid at arbitrary temperatures for ratios $R$ typically $R \ll 1$ [5-7].

\section{Theory}

Because the Fermi energy is one of the variables for calculating line shapes in the Raman spectra from semiconductors, we first present in Sec. 2.1 the theory for the BGN and PDOS models by which numerical methods give closed-form analytic expressions that relate carrier concentrations to Fermi energy $E_{\mathrm{F}}$, temperature $T$, and donor dopant densities $N_{\mathrm{D}}$. Then, within the context of these BGN and PDOS models, we develop in Sec. 2.2 the theory for calculating the line shapes observed in Raman spectra.

\subsection{Fermi Energy and Electron Density}

The temperature and donor dopant density are the independent input parameters for evaluating the Raman line shape factor given in Eq. (19). The Fermi energy depends in turn on the dopant density, in this case the donor density $N_{\mathrm{D}}$ and $T$. For given $N_{\mathrm{D}}$ and $T$, we present here models to compute the Fermi energy with full Fermi-Dirac statistics for the carriers at finite temperature. By using these models, we calculate the majority electron density as a function of the Fermi energy in zinc blende, n-type GaAs for donor densities between $10^{16} \mathrm{~cm}^{-3}$ and $10^{19} \mathrm{~cm}^{-3}$. These calculations solve the charge neutrality equation self-consistently in terms of two main classes of models: the bandgap narrowing (BGN) model and three different parabolic densities of states (PDOS) models.

Some researchers propose that Raman spectra may be a way to determine temperatures with spatial resolutions across wafers on the order of micrometers when the Fermi energy and dopant density are known from other independent measurements. Comparing the results given by the BGN and PDOS models, which are described below, also provides predictions on the sensitivity of the Fermi energy and Raman spectrum to variations in temperature.

\subsubsection{BGN Model}

The bandgap narrowing (BGN) model is a two-band model with one equivalent conduction band and one equivalent valence band at the $\Gamma$ point in Brilluoin space. The BGN model is related to earlier work on ntype GaAs [9] and includes modifications to the densities of states due to high concentrations of dopants, bandgap narrowing, and many-body effects associated with carrier-carrier interactions (carrier-carrier exchange and correlation). This BGN model is fully self-consistent and uses the Klauder self-energy (fifth 
level of approximation) to calculate the distorted-perturbed densities of states for the carriers. This BGN model has the following main features:

1) Many-body quantum effects of carrier-carrier interactions and carrier-dopant ion interactions, bandgap narrowing, and distorted-perturbed densities of states for the carriers [9],

2) Iterative and self-consistent solutions of the coupled charge neutrality equation and Klauder's fifth level of approximation for the renormalized self-energy propagator from which the distorted-perturbed densities of states are calculated,

3) Full Fermi-Dirac statistics for the carriers at finite temperature, and

4) Statistical analyses to give closed-form analytic expressions from very large, calculated data sets for carrier densities as functions of the Fermi energy.

Tables 1 and 2 contain the input parameters for the BGN calculations in Ref. [9] and for the BGN model given here.

The electron $n$ and hole $h$ concentrations in units of $\mathrm{cm}^{-3}$ at thermal equilibrium are given, respectively, by

$n=\int_{-\infty}^{+\infty} f_{0}(E) \rho_{\mathrm{C}}(E) \mathrm{d} E$ and $h=\int_{-\infty}^{+\infty}\left[1-f_{0}(E)\right] \rho_{\mathrm{V}}(E) \mathrm{d} E$, where $\rho_{\mathrm{C}}(E)$ and $\rho_{\mathrm{V}}(E)$ are, respectively, the electron density of states for the equivalent conduction band and the hole density of states for the equivalent valence band [9], where $f_{0}(E)=\left\{1+\exp \left[\left(E-E_{\mathrm{F}}\right) / k_{\mathrm{B}} T\right]\right\}^{-1}$ is the Fermi-Dirac distribution function. The calculations incorporate the Thomas-Fermi expression for the screening radius,

$$
r_{\mathrm{s}}^{2}=-\frac{4 \pi e^{2}}{\varepsilon \varepsilon_{0}} \int_{-\infty}^{+\infty} \frac{\mathrm{d} f_{0}(E)}{\mathrm{d} E}\left[\rho_{\mathrm{C}}(E)-\rho_{\mathrm{V}}(E)\right] \mathrm{d} E,
$$

and the charge neutrality condition

$$
N_{\mathrm{I}}=n-h,
$$

to compute self-consistently the Fermi energy $E_{\mathrm{F}}$ and the screening radius $r_{\mathrm{s}}$ for given values of the ionized dopant concentration $N_{\mathrm{I}}$ and temperature $T$. The static dielectric constant is $\varepsilon$ and the permittivity of free space is $\varepsilon_{0}$. The ionized dopant concentration is positive for ntype material (donor ions) and negative for p-type material (acceptor ions). The results reported here are for n-type material. For the case discussed here, $N_{\mathrm{I}}=$ $N_{\mathrm{D}}$. The results for the screening radius $r_{\mathrm{s}}$ are not reported here because they are not needed to extract carrier concentrations from Raman scattering measurements.

Table 1. Fundamental Constants

\begin{tabular}{llll}
\hline \hline Parameter & Symbol & Value & Units \\
\hline Planck's constant & $\hbar$ & $6.5836 \times 10^{-16}$ & $\mathrm{eV} \cdot \mathrm{s}$ \\
Boltzmann's constant & $k_{\mathrm{B}}$ & $8.6174 \times 10^{-5}$ & $\mathrm{eV} / \mathrm{K}$ \\
electron rest mass & $m_{0}$ & $9.1072 \times 10^{-28}$ & $\mathrm{~g}$ \\
electronic charge & $e$ & $-4.802 \times 10^{-10}$ & $\mathrm{esu}$ \\
Bohr radius & $a_{\mathrm{B}}$ & $0.5291 \times 10^{-8}$ & $\mathrm{~cm}$ \\
energy associated with 1 Rydberg & $e^{2} / 2 a_{\mathrm{B}}$ & 13.6 & $\mathrm{eV}$ \\
speed of light & $c$ & $2.9979 \times 10^{10}$ & $\mathrm{~cm} / \mathrm{s}$ \\
wave length associated with 1 eV & {$\left[\lambda_{0}\right]$} & $1.2396 \times 10^{-4}$ & $\mathrm{~cm}$ \\
wave number associated with 1 eV & {$\left[k_{0}\right]$} & $8.0668 \times 10^{3}$ & $\mathrm{~cm}$ \\
dielectric constant in vacuum & $\varepsilon$ & $8.854 \times 10^{-12}$ & $\mathrm{~F} / \mathrm{m}$ \\
\hline
\end{tabular}

Table 2. BGN model input parameters for intrinsic zinc blende GaAs at $300 \mathrm{~K}$. The energies of the extrema of the conduction and valence sub-bands are referenced to the bottom of the conduction sub-band at the $\Gamma$ symmetry point in the Brillouin zone of the reciprocal lattice space. The mass of the free electron is $m_{0}$. These GaAs data are from Ref. [12].

\begin{tabular}{llll}
\hline \hline Parameter & Symbol & Value & Units \\
\hline bandgap & $E_{\mathrm{G}}=\left|-E_{\mathrm{v} \Gamma}\right|$ & 1.424 & $\mathrm{eV}$ \\
effective mass for conduction band (2-band model) density of states & $m_{\mathrm{C}}$ & 0.067 & $m_{0}$ \\
effective mass for valence band (2-band model) density of states & $m_{\mathrm{V}}$ & 0.572 & $m_{0}$ \\
number of equivalent conduction bands & $N_{\mathrm{C}}$ & 1 & \\
number of equivalent valence bands & $N_{\mathrm{V}}$ & 1 & \\
\hline
\end{tabular}




\subsubsection{PDOS Models}

The PDOS models use parabolic densities of states for all equivalent bands and sub-bands. Unlike the BGN model in Ref. [9], PDOS models such as the fourband PDOS model for GaSb in Ref. [10] do not include modifications to the densities of states due to manybody effects and high concentrations of dopants and carriers because of computational limitations associated with treating a four-band model in the context of the Klauder self-energy method (fifth level of approximation).

The zero of energy is at the minimum energy value (bottom) of the conduction $\Gamma$ sub-band, $E_{\mathrm{c} \Gamma 0}=0.0$. The bottoms of the conduction $\mathrm{L}$ and $\mathrm{X}$ sub-bands are, respectively, at $E_{\mathrm{cL}}$ and $E_{\mathrm{cX}}$. The maximum energy value (top) of the degenerate valence $\Gamma$ sub-band is $-E_{\mathrm{G}}$, where $E_{\mathrm{G}}$ is the intrinsic bandgap of GaAs. The split-off valence sub-band at $\Gamma$ due to spin-orbit coupling is neglected. The probabilities for typical holes in equilibrium to occupy appreciably these states in the split-off valence sub-band at $\Gamma$ are very low. This means that the Fermi energies should be sufficiently above the valence sub-band maximum at $\Gamma$. Placing exact limits on the Fermi energies for which the PDOS models are valid would be tenuous, because knowledge of how the various sub-bands move relative to one another due to the dopant concentrations considered here and due to many body effects is not adequate. Table 3 lists the input parameters for the PDOS models.

The heavy hole mass $m_{\mathrm{hh}}$ and light hole mass $m_{\mathrm{lh}}$ for the two degenerate sub-bands at the top of the valence band are combined to give an effective mass

$$
m_{\mathrm{v} \tilde{\AA}}=\left(m_{\mathrm{hh}}^{3 / 2}+m_{\mathrm{lh}}^{3 / 2}\right)^{2 / 3},
$$

for the valence topmost sub-band, which becomes the equivalent valence band with a hole energy dispersion given by $E_{\mathrm{v} \Gamma}(\boldsymbol{k}) \approx-E_{\mathrm{G}}-\left(\hbar^{2} k^{2} / 2 m_{\mathrm{v} \Gamma} m_{0}\right)$.

The general expression for the parabolic densities of states for electrons and holes per band extrema and per spin direction is given by

$$
\rho(E)=\frac{N_{\mathrm{e}} 4 \pi V \sqrt{E}}{\left(8 \pi^{3}\right)\left(\hbar^{2} / 2 m^{*} m_{0}\right)^{3 / 2}},
$$

where $N_{\mathrm{e}}$ is the number of equivalent ellipsoids in the first Brillouin zone, the volume of the unit cell is $V=$ $a_{\mathrm{L}}{ }^{3}, a_{\mathrm{L}}$ is the lattice constant, $m^{*}$ is one of the effective masses listed in Tables 2 and 3 for the appropriate band extrema, and $m_{0}$ is the free electron mass.

We sub-divide the PDOS models into the PDOS2, PDOS2NPG, and PDOS4 models. All three PDOS models include the equivalent valence band described by Eq. (4).

Table 3. PDOS model input parameters for intrinsic zinc blende GaAs at $300 \mathrm{~K}$. The energies of the extrema of the conduction and valence subbands are referenced to the bottom of the conduction sub-band at the $\Gamma$ symmetry point in the Brillouin zone of the reciprocal lattice space. The

\begin{tabular}{|c|c|c|c|}
\hline Parameter & Symbol & Value & Units \\
\hline bandgap & $E_{\mathrm{G}}=\left|-E_{\mathrm{v} \Gamma}\right|$ & 1.424 & $\mathrm{eV}$ \\
\hline bottom of the conduction L sub-band & $E_{\mathrm{cL}}$ & 0.29 & $\mathrm{eV}$ \\
\hline bottom of the conduction $\mathrm{X}$ sub-band & $E_{\mathrm{vX}}$ & 0.48 & $\mathrm{eV}$ \\
\hline top of the degenerate valence $\Gamma$ sub-band & $-E_{\mathrm{v} \Gamma}$ & 1.424 & $\mathrm{eV}$ \\
\hline spin-orbit splitting & $-E_{\mathrm{So}}$ & 0.34 & $\mathrm{eV}$ \\
\hline top of the split-off (spin-orbit splitting) valence $\Gamma$ sub-band & $-E_{\mathrm{so} \Gamma}=-E_{\mathrm{v} \Gamma}-E_{\mathrm{so}}$ & 1.764 & $\mathrm{eV}$ \\
\hline effective mass of conduction $\Gamma$ sub-band & $m_{\mathrm{c} \Gamma}$ & 0.063 & $m_{0}$ \\
\hline non-parabolicity factor (quartic term prefactor) for conduction $\Gamma$ sub-band & $\xi$ & 0.824 & \\
\hline transverse L sub-band mass & $m_{\mathrm{tL}}$ & 0.075 & $m_{0}$ \\
\hline longitudinal L sub-band mass & $m_{\mathrm{IL}}$ & 1.9 & $m_{0}$ \\
\hline effective mass of conduction L sub-band & $m_{\mathrm{cL}}=\left(m_{\mathrm{lL}} m_{\mathrm{tL}}^{2}\right)^{1 / 3}$ & 0.222 & $m_{0}$ \\
\hline transverse $\mathrm{X}$ sub-band mass & $m_{\mathrm{tX}}$ & 0.19 & $m_{0}$ \\
\hline longitudinal X sub-band mass & $m_{\mathrm{IX}}$ & 1.9 & $m_{0}$ \\
\hline effective mass of conduction $\mathrm{X}$ sub-band & $m_{\mathrm{cX}}=\left(m_{\mathrm{lX}} m_{\mathrm{tX}}^{2}\right)^{1 / 3}$ & 0.409 & $m_{0}$ \\
\hline light hole mass of valence $\Gamma$ sub-band & $m_{\mathrm{lh}}$ & 0.082 & $m_{0}$ \\
\hline heavy hole mass of valence $\Gamma$ sub-band & $m_{\mathrm{hh}}$ & 0.51 & $m_{0}$ \\
\hline effective mass of valence $\Gamma$ sub-band & $m_{\mathrm{v} \Gamma}$ & 0.53 & $m_{0}$ \\
\hline splitoff band mass of the valence sub-band at $\Gamma$ & $m_{\mathrm{so}}$ & 0.15 & $m_{0}$ \\
\hline number of equivalent conduction $\mathrm{L}$ sub-bands & $N_{\mathrm{cL}}$ & 4 & \\
\hline number of equivalent conduction $\mathrm{X}$ sub-bands & $N_{\text {cX }}$ & 3 & \\
\hline
\end{tabular}
mass of the free electron is $m_{0}$. These GaAs data are from Refs. [11,12]. 


\section{PDOS2 Model}

The PDOS2 model uses one equivalent conduction band and one equivalent valence band at the $\Gamma$ symmetry point in the Brilluoin space for the integrals that appear in Eqs. (1) to (3). The electron energy dispersion for the equivalent conduction band is $E_{\mathrm{c} \Gamma}(\boldsymbol{k}) \approx E_{\mathrm{c} \Gamma 0}+$ $\left(\hbar^{2} k^{2} / 2 m_{\mathrm{C}} m_{0}\right)$.

\section{PDOS2NPG Model}

The PDOS2NPG model is a two-band model with one equivalent conduction band and one equivalent valence band at the $\Gamma$ point in Brilluoin space. It has no bandgap narrowing, but it includes the non-parabolicity for the electron energy dispersion in the equivalent conduction band at $\Gamma$. According to Ref. [11], we may include non-quadratic $|\boldsymbol{k}|^{l}$ terms in the electron energy dispersion $E_{\mathrm{c} \Gamma}(\boldsymbol{k})$ for the conduction $\Gamma$ sub-band in GaAs when $\boldsymbol{k}$ is small, namely,

$$
E_{\mathrm{c} \Gamma}(\boldsymbol{k}) \approx E_{\mathrm{c} \Gamma 0}+\left(\hbar^{2} k^{2} / 2 m_{\mathrm{C}} m_{0}\right)+\left(\xi / E_{\mathrm{G}}\right)\left(\hbar^{2} k^{2} / 2 m_{\mathrm{C}} m_{0}\right)^{2},
$$

where $\xi$ is the non-parabolicity factor. We use the Kane three level $\boldsymbol{k} \cdot \boldsymbol{p}$ model [11], which does not include the conduction sub-bands at $\mathrm{L}$ and $\mathrm{X}$, to include quartic terms in $E(\boldsymbol{k})$ with $l=4$.

\section{PDOS4 Model}

The PDOS4 model has three conduction sub-bands at the respective $\Gamma, \mathrm{L}$, and $\mathrm{X}$ symmetry points in the Brilluoin space and one equivalent valence band at the $\Gamma$ symmetry point. For the PDOS4, we modify here the PDOS model for GaSb in Ref. [10] so that it is valid for GaAs. It uses the parabolic electron energy dispersion $E_{\mathrm{c \Gamma}}(\boldsymbol{k})$ for the conduction $\Gamma$ sub-band in GaAs when $\boldsymbol{k}$ is small, namely, $E_{\mathrm{c} \Gamma}(\boldsymbol{k}) \approx E_{\mathrm{c} \Gamma 0}+\left(\hbar^{2} k^{2} / 2 m_{\mathrm{C}} m_{0}\right)$. The general expression for the temperature dependence of conduction sub-band minima relative to the top of the valence band at $\Gamma$ is given by [12],

$$
E_{i}=E_{i 0}-\left[A_{i} T^{2} /\left(T+B_{i}\right)\right]
$$

in units of $\mathrm{eV}$, where $i=\Gamma$, $\mathrm{L}$, or $\mathrm{X}$. The values for the coefficients $E_{i 0}, A_{i}$, and $B_{i}$ are listed in Table 4. Because 8 permutations of the wave vector in the (111) direction exist, there are $8 \mathrm{~L}$ sub-band ellipsoids with centers located near the boundary of the first Brillouin zone. Also, because 6 permutations of the wave vector in the (100) direction exist, there are $6 \mathrm{X}$ sub-band ellipsoids
Table 4. Coefficients for the temperature dependence of the conduction band extrema that are used in Eq. (15). These data are from Ref. [12].

\begin{tabular}{llll}
\hline \hline Parameter & Symbol & Value & Units \\
\hline Г sub-band coefficients & $E_{\Gamma 0}$ & 1.519 & $\mathrm{eV}$ \\
& $A_{\Gamma}$ & $5.405 \times 10^{-4}$ & $\mathrm{eV} / \mathrm{K}$ \\
& $B_{\Gamma}$ & 204 & $\mathrm{~K}$ \\
& & & \\
L sub-band coefficients & $E_{\mathrm{L} 0}$ & 1.815 & $\mathrm{eV}$ \\
& $A_{\mathrm{L}}$ & $6.05 \times 10^{-4}$ & $\mathrm{eV} / \mathrm{K}$ \\
& $B_{\mathrm{L}}$ & 204 & $\mathrm{~K}$ \\
& & & \\
X sub-band coefficients & $E_{\mathrm{X} 0}$ & 1.981 & $\mathrm{eV}$ \\
& $A_{\mathrm{X}}$ & $4.60 \times 10^{-4}$ & $\mathrm{eV} / \mathrm{K}$ \\
& $B_{\mathrm{X}}$ & 204 & $\mathrm{~K}$ \\
\hline
\end{tabular}

with centers located near the boundary of the first Brillouin zone. Since about half of each ellipsoid is in the neighboring zone, the number of equivalent subbands $N_{\mathrm{cL}}$ for the $E_{\mathrm{cL}}$ is four, and the number of equivalent sub-bands $N_{\mathrm{cX}}$ for the X sub-band is three.

In terms of the four-band PDOS4 model for room temperature n-type GaAs, the total density of states $\rho_{\mathrm{c}}(E)$ for the majority carrier electrons in n-type GaAs then becomes

$$
\rho_{\mathrm{c}}(E)=\rho_{\mathrm{c} \Gamma}(E)+\rho_{\mathrm{cL}}(E)+\rho_{\mathrm{cX}}(E),
$$

where $\rho_{\mathrm{c \Gamma}}(E), \rho_{\mathrm{cL}}(E)$, and $\rho_{\mathrm{cX}}(E)$ are the sub-band densities of states for the conduction $\Gamma$, L, and X sub-bands with effective masses of $m_{\mathrm{c} \Gamma}, m_{\mathrm{cL}}$, and $m_{\mathrm{cX}}$, respectively. The density of states for the minority carrier holes is

$$
\rho_{\mathrm{v}}(E)=\rho_{\mathrm{v \Gamma}}(E)
$$

with an effective mass of $m_{v \Gamma}$.

\subsection{Dielectric Response Function}

The longitudinal optical (LO) phonons and plasmons interact in polar semiconductors such as GaAs to form LO phonon-plasmon modes. The theoretical line shape function $L_{\mathrm{A}}(\boldsymbol{q}, \omega)$ of the Raman spectrum due to longitudinal optical (LO) phonon-plasmon coupled modes is then given for the configuration to which this theory will be applied by [8]

$$
L_{\mathrm{A}}(\boldsymbol{q}, \omega)=\left(1-e^{-\hbar \omega / k_{\mathrm{B}} T}\right)^{-1}\left(\frac{\omega_{0}^{2}-\omega^{2}}{\omega_{\mathrm{TO}}^{2}-\omega^{2}}\right)^{2} \operatorname{Im}\left\{-\frac{1}{\varepsilon(\boldsymbol{q}, \omega)}\right\},
$$


where $\boldsymbol{q}$ is the scattering wave vector, $\omega$ is the Raman angular frequency shift, $\hbar$ is the Planck constant, $k_{\mathrm{B}}$ is the Boltzmann constant, $T$ is the temperature in Kelvin, $\omega_{0}=\omega_{\mathrm{TO}}\left(1+C_{\mathrm{FH}}\right)^{1 / 2}$ is a parameter with the dimensions of angular frequency, $C_{\mathrm{FH}}$ is the dimensionless FaustHenry coefficient which includes the LO/transverse optical (TO) phonon scattering ratio, and $\omega_{\mathrm{TO}}$ is the TO phonon angular frequency. Table 5 contains values for the parameters in Eq. (10). The total longitudinal dielectric response function $\varepsilon(\boldsymbol{q}, \omega)$ in the random phase approximation (RPA) is described by

$$
\varepsilon(\boldsymbol{q}, \omega)=1+4 \pi \chi_{\mathrm{VE}}+4 \pi \chi_{\mathrm{L}}(\omega)+4 \pi \chi_{\mathrm{e}}(\boldsymbol{q}, \omega),
$$

where the dielectric susceptibility $\chi_{\mathrm{VE}}$ is the contribution from valence electrons, $\chi_{\mathrm{L}}(\omega)$ is the contribution from the polar lattice phonons, and $\chi_{\mathrm{e}}(\boldsymbol{q}, \omega)$ is the contribution from the conduction electrons. The high frequency dielectric constant $\varepsilon_{\infty}$ is defined to be $\varepsilon_{\infty}=1+$ $4 \pi \chi_{\mathrm{VE}}$. Equation (11) then becomes for a binary semiconductor,

$$
\varepsilon(\boldsymbol{q}, \omega)=\varepsilon_{\infty}+4 \pi \chi_{\mathrm{L}}(\omega)+4 \pi \chi_{\mathrm{e}}(\boldsymbol{q}, \omega) .
$$

The contribution of the polar lattice is given by [8]

$$
4 \pi \chi_{\mathrm{L}}(\omega)=\varepsilon_{\infty}\left(\frac{\omega_{\mathrm{LO}}^{2}-\omega_{\mathrm{TO}}^{2}}{\omega_{\mathrm{TO}}^{2}-\omega^{2}}\right),
$$

when phonon damping may be neglected and where $\omega_{\mathrm{LO}}$ is the LO phonon angular frequency.

Within the context of the RPA, the Lindhard expression [13] gives the electronic contribution to the dielec- tric response function $4 \pi \chi_{\mathrm{e}}^{0}(\boldsymbol{q}, \omega)$ that describes light scattering by the conduction electrons in doped semiconductors. We introduce the collision relaxation time $\tau$ that describes the losses associated with electronphonon and electron-dopant interactions and the corresponding angular collision frequency $\Gamma=\tau^{-1}$. Mermin [14] showed that replacing $\omega$ with $\omega+i \Gamma$ in the Lindhard expression for $4 \pi \chi_{\mathrm{e}}^{0}(\boldsymbol{q}, \omega)$ fails to conserve the number of local electrons and therefore is not the correct way to include collision broadening in $4 \pi \chi_{\mathrm{e}}^{0}(\boldsymbol{q}, \omega)$. Instead, Mermin assumed that within the framework of a relaxation time approximation the electron-phonon and electron-dopant interactions relax the electron density matrix to a local equilibrium density matrix $[4,14]$. He then obtained the following Lindhard-Mermin relation [14] for $\chi_{\mathrm{e}}(\boldsymbol{q}, \omega)$,

$$
\chi_{\mathrm{e}}(\boldsymbol{q}, \omega)=\frac{(\omega+i \Gamma) \chi_{\mathrm{e}}^{0}(\boldsymbol{q}, 0) \chi_{\mathrm{e}}^{0}(\boldsymbol{q}, \omega+i \Gamma)}{\omega \chi_{\mathrm{e}}^{0}(\boldsymbol{q}, 0)+i \Gamma \chi_{\mathrm{e}}^{0}(\boldsymbol{q}, \omega+i \Gamma)}
$$

where the Lindhard expression $\chi_{\mathrm{e}}^{0}(\boldsymbol{q}, \omega+i \Gamma)$ for electrons occupying states in a single equivalent conduction band is given by,

$$
\begin{aligned}
& 4 \pi \chi_{\mathrm{e}}^{0}(\boldsymbol{q}, \omega+i \Gamma)=\frac{e^{2}}{\pi^{2} q^{2}} \int f_{0}(E) \\
& \times\left[\left(\frac{\hbar^{2} q^{2}}{2 m_{\mathrm{C}} m_{0}}+\frac{\hbar^{2} \boldsymbol{q} \cdot \boldsymbol{k}}{m_{\mathrm{C}} m_{0}}-\hbar(\omega+i \Gamma)\right)^{-1}\right. \\
& \left.+\left(\frac{\hbar^{2} q^{2}}{2 m_{\mathrm{C}} m_{0}}-\frac{\hbar^{2} \boldsymbol{q} \cdot \boldsymbol{k}}{m_{\mathrm{C}} m_{0}}+\hbar(\omega+i \Gamma)\right)^{-1}\right] \mathrm{d}^{3} \boldsymbol{k},
\end{aligned}
$$

\begin{tabular}{|c|c|c|c|}
\hline Parameter & Symbol & Value & Units \\
\hline lattice constant & $a_{\mathrm{L}}$ & $5.65 \times 10^{-8}$ & $\mathrm{~cm}$ \\
\hline static dielectric constant & $\varepsilon_{0}$ & 13.1 & \\
\hline high frequency dielectric constant & $\varepsilon_{\infty}$ & 10.9 & \\
\hline longitudinal optical (LO) phonon energy & $\hbar \omega_{\mathrm{LO}}$ & $\begin{array}{l}0.0353 \\
285\end{array}$ & $\begin{array}{l}\mathrm{eV} \\
\mathrm{cm}^{-1}\end{array}$ \\
\hline transverse optical (TO) phonon energy & $\hbar \omega_{\mathrm{TO}}$ & $\begin{array}{l}0.0332 \\
268\end{array}$ & $\begin{array}{l}\mathrm{eV} \\
\mathrm{cm}^{-1}\end{array}$ \\
\hline $\begin{array}{l}\text { Energy associated with the angular collision frequency } \Gamma \\
\text { due to electron-phonon and electron-dopant ion interactions }\end{array}$ & $\hbar \Gamma$ & $\begin{array}{l}\sim 0.0124 \\
\sim 100\end{array}$ & $\begin{array}{l}\mathrm{eV} \\
\mathrm{cm}^{-1}\end{array}$ \\
\hline Faust-Henry coefficient & $C_{\mathrm{FH}}$ & -0.4 & \\
\hline $\begin{array}{l}\text { effective mass for the single equivalent conduction } \\
\text { band density of states in Eq. (6) }\end{array}$ & $m_{\mathrm{C}}$ & 0.067 & $m_{0}$ \\
\hline
\end{tabular}

Table 5. Dielectric response function input parameters for intrinsic zinc blende GaAs at $300 \mathrm{~K}$. The energies of the extrema of the conduction and valence sub-bands are referenced to the bottom of the conduction subband at the $\Gamma$ symmetry point in the Brillouin zone of the reciprocal lattice space. The mass of the free electron is $m_{0}$. These GaAs data are from Ref. [14]. 
where $f_{0}(E)=\left\{1+\exp \left[\left(E-E_{\mathrm{F}}\right) / k_{\mathrm{B}} T\right]\right\}^{-1}$ is the FermiDirac distribution function, $E_{\mathrm{F}}$ is the Fermi energy in $\mathrm{eV}$, and $T$ is the temperature in Kelvin.

The integrand in Eq. (15) is independent of the azimuthal angle $\varphi$ so that,

$$
\int_{0}^{\infty} k^{2} \mathrm{~d} k \int_{0}^{\pi} \sin \theta \mathrm{d} \theta \int_{0}^{2 \pi} \mathrm{d} \varphi(\ldots)=2 \pi \int_{0}^{\infty} k^{2} \mathrm{~d} k \int_{-1}^{1} \mathrm{~d} \mu(\ldots)
$$

where $\mu=\cos \theta, \mathrm{d} \mu=-\sin \theta$, and $\boldsymbol{q} \cdot \boldsymbol{k}=q k \mu$. To simplify Eq. (15) further, we introduce the following dimensionless normalized quantities: $Q=q a_{\mathrm{B}}, K=k a_{\mathrm{B}}, K(E)=$ $\sqrt{\left(2 m_{0} m_{\mathrm{C}} a_{\mathrm{B}}{ }^{2} / \hbar^{2}\right) E}$, and $\Omega^{2}=\left\{\hbar(\omega+i \Gamma) m_{\mathrm{C}} / 13.6 \mathrm{eV}\right\}$ where $\left(e^{2} / 2 a_{B}\right)=\left(\hbar^{2} / 2 m_{0} a_{\mathrm{B}}^{2}\right)=13.6 \mathrm{eV}$. Equation (15) then becomes with these definitions the following expression,

$$
\begin{aligned}
& 4 \pi \chi_{\mathrm{e}}{ }^{0}(\boldsymbol{q}, \omega+i \Gamma) \\
& =\frac{4 m_{\mathrm{C}}}{\pi Q^{2}} \int_{0}^{\infty} K^{2} \mathrm{~d} K \\
& \int_{-1}^{1} \mathrm{~d} \mu\left(1+\exp \left[\left\{\left(13.6 \mathrm{eV} \mathrm{K} / m_{\mathrm{C}}\right)-E_{\mathrm{F}}\right\} / k_{\mathrm{B}} T\right]\right)^{-1} \\
& \times\left[\left(Q^{2}+2 Q K \mu-\Omega^{2}\right)^{-1}+\left(Q^{2}-2 Q K \mu+\Omega^{2}\right)^{-1}\right] .
\end{aligned}
$$

Performing analytically the angular integration over $\mu$ gives the complex results

$$
\begin{aligned}
& 4 \pi \chi_{\mathrm{e}}^{0}(\boldsymbol{q}, \omega+i \Gamma) \\
& =\frac{2 m_{\mathrm{C}}}{\pi Q^{3}} \int_{0}^{\infty} K \mathrm{~d} K\left(1+\exp \left[\left\{\left(13.6 \mathrm{eV} \mathrm{K} K^{2} m_{\mathrm{C}}\right)-E_{\mathrm{F}}\right\} / k_{\mathrm{B}} T\right]\right)^{-1} \\
& \times\left[\ln \left(\frac{\rho_{+}^{2}+\gamma^{2}}{\rho_{-}^{2}+\gamma^{2}}\right)+\ln \left(\frac{\tau_{+}^{2}+\gamma^{2}}{\tau_{-}^{2}+\gamma^{2}}\right)+i\left(\eta_{+}-\eta_{-}\right)+i\left(\sigma_{+}-\sigma_{-}\right)\right]
\end{aligned}
$$

where $\rho_{ \pm}=Q^{2} \pm 2 Q K-\alpha, \tau_{ \pm}=Q^{2} \pm 2 Q K+\alpha$, $\eta_{ \pm}=-\operatorname{sign}\left(\rho_{ \pm}\right) \arctan \left(\gamma / \rho_{ \pm}\right)-(\pi / 2)\left[1-\operatorname{sign}\left(\rho_{ \pm}\right)\right]$, $\sigma_{ \pm}=+\operatorname{sign}\left(\tau_{ \pm}\right) \arctan \left(\gamma / \tau_{ \pm}\right)+(\pi / 2)\left[1-\operatorname{sign}\left(\tau_{ \pm}\right)\right]$, $\Omega^{2}=\alpha+i \gamma, \alpha=\left(\hbar \omega m_{\mathrm{C}} / 13.6 \mathrm{eV}\right), \gamma=\left(\hbar \Gamma m_{\mathrm{C}} / 13.6 \mathrm{eV}\right)$, and $-\pi \leq \arctan (\psi) \leq \pi$.

When the temperature $T=0$, the Fermi function $f_{0}(E)=\left(1+\exp \left[\left\{\left(13.6 \mathrm{eV} \mathrm{K} K^{2} / m_{\mathrm{C}}\right)-E_{\mathrm{F}}\right\} / k_{\mathrm{B}} T\right]\right)^{-1}$ is the unit step function. Then, the integral in Eq. (17) $\int_{0}^{\infty} K^{2} \mathrm{~d} K(\ldots)$ is $\int_{0}^{K_{\mathrm{F}}} K^{2} \mathrm{~d} K(\ldots)$, and an analytic evaluation is possible [4], where $K_{\mathrm{F}}=\sqrt{m_{\mathrm{C}} E_{\mathrm{F}} / 13.6 \mathrm{eV}}$. But, when $T$ is room temperature, analytic evaluations are not possible. Researchers approximated the integral $\int_{0}^{\infty} K^{2} \mathrm{~d} K(\ldots)$ at finite temperatures by expanding its integrand in terms of the ratio $R=\left(Q^{2} / \alpha\right)$ for either very small or large $R$. However, such expansions do not capture all of the spectral information in Eq. (17).
The theoretical line shape function $L_{\mathrm{A}}(\boldsymbol{q}, \omega)$ of the Raman spectrum then becomes

$L_{\mathrm{A}}(\boldsymbol{q}, \omega)$

$=\left(1-e^{-\hbar \omega / k_{\mathrm{B}} T}\right)^{-1}\left(\frac{\omega_{0}^{2}-\omega^{2}}{\omega_{\mathrm{TO}}^{2}-\omega^{2}}\right)^{2}\left\{\frac{\varepsilon_{\mathrm{i}}(\boldsymbol{q}, \omega)}{\varepsilon_{\mathrm{r}}^{2}(\boldsymbol{q}, \omega)+\varepsilon_{\mathrm{i}}^{2}(\boldsymbol{q}, \omega)}\right\}$,

where $\operatorname{Im}\left\{-\frac{1}{\varepsilon(\boldsymbol{q}, \omega)}\right\}=\left\{\frac{\varepsilon_{\mathrm{i}}(\boldsymbol{q}, \omega)}{\varepsilon_{\mathrm{r}}^{2}(\boldsymbol{q}, \omega)+\varepsilon_{\mathrm{i}}^{2}(\boldsymbol{q}, \omega)}\right\}$,

$\varepsilon_{\mathrm{r}}(\boldsymbol{q}, \omega)=\operatorname{Re}\{\varepsilon(\boldsymbol{q}, \omega)\}=\varepsilon_{\infty}\left\{\frac{\omega_{\mathrm{LO}}{ }^{2}-\omega_{\mathrm{TO}}{ }^{2}}{\omega_{\mathrm{TO}}{ }^{2}-\omega^{2}}\right\}$

$+4 \pi \chi_{\mathrm{re}}(\boldsymbol{q}, \omega+i \Gamma)$, and

$\varepsilon_{\mathrm{i}}(\boldsymbol{q}, \omega)=\operatorname{Im}\{\varepsilon(\boldsymbol{q}, \omega)\}=4 \pi \chi_{\text {ie }}(\boldsymbol{q}, \omega+i \Gamma)$.

The Lindhard-Mermin relation Eq. (14) then gives expressions for $\chi_{\mathrm{re}}(\boldsymbol{q}, \omega+i \Gamma)=\operatorname{Re}\left\{\chi_{\mathrm{e}}(\boldsymbol{q}, \omega+i \Gamma)\right\}$ and $\chi_{\mathrm{ie}}(\boldsymbol{q}, \omega+i \Gamma)=\operatorname{Im}\left\{\chi_{\mathrm{e}}(\boldsymbol{q}, \omega+i \Gamma)\right\}$ as functions of $\chi_{\mathrm{re}}^{0}(\boldsymbol{q}, \omega+i \Gamma)=\operatorname{Re}\left\{\chi_{\mathrm{e}}^{0}(\boldsymbol{q}, \omega+i \Gamma)\right\}$ and $\chi_{\mathrm{ie}}{ }^{0}(\boldsymbol{q}, \omega+i \Gamma)$ $=\operatorname{Im}\left\{\chi_{\mathrm{e}}^{0}(\boldsymbol{q}, \omega+i \Gamma)\right\}$. Even though the factors in Eqs. (10) and (18) give the contributions to the Raman line shape for each scattering mechanism, an examination of only the factor

$$
\begin{aligned}
& \operatorname{Im}\left\{-\frac{1}{\varepsilon(\boldsymbol{q}, \omega)}\right\}=\left\{\frac{\varepsilon_{i}(\boldsymbol{q}, \omega)}{\varepsilon_{\mathrm{r}}^{2}(\boldsymbol{q}, \omega)+\varepsilon_{\mathrm{i}}^{2}(\boldsymbol{q}, \omega)}\right\} \\
& =\left\{\frac{\left(\omega_{\mathrm{TO}}^{2}-\omega^{2}\right)^{2} 4 \pi \chi_{\mathrm{ie}}(\boldsymbol{q}, \omega+i \Gamma)}{D_{\mathrm{r}}^{2}(\boldsymbol{q}, \omega+i \Gamma)}\right\}
\end{aligned}
$$

gives the spectral information for extracting the Fermi energy. The denominator in Eq. (19) is given by the expression,

$$
\begin{aligned}
& D_{\mathrm{r}}^{2}(\boldsymbol{q}, \omega+i \Gamma)=\varepsilon_{\infty}{ }^{2}\left(\omega_{\mathrm{LO}}{ }^{2}-\omega_{\mathrm{TO}}{ }^{2}\right)^{2} \\
& +2 \varepsilon_{\infty}\left(\omega_{\mathrm{LO}}{ }^{2}-\omega^{2}\right)\left(\omega_{\mathrm{TO}}{ }^{2}-\omega^{2}\right) 4 \pi \chi_{\mathrm{ie}}(\boldsymbol{q}, \omega+i \Gamma) \\
& +\left(\omega_{\mathrm{TO}}{ }^{2}-\omega^{2}\right)^{2}\left[\left\{4 \pi \chi_{\mathrm{re}}(\boldsymbol{q}, \omega+i \Gamma)\right\}^{2}\right. \\
& \left.+\left\{4 \pi \chi_{\mathrm{ie}}(\boldsymbol{q}, \omega+i \Gamma)\right\}^{2}\right] .
\end{aligned}
$$

Section 3 below contains the procedures for extracting electron densities from the Fermi energies.

\section{Numerical Results - Electron Density and Fermi Energy}

Because the Fermi energy is one of the variables for calculating the Raman line shape, we give the numeri- 
cal results for calculating the Fermi energy in terms of the BGN and PDOS models and the analytic expressions that relate the carrier concentrations to the Fermi energy.

\subsection{BGN Model}

We solve self-consistently, by means of an iterative procedure, Eq. (3) with the distorted-perturbed carrier densities of states $\rho_{\mathrm{C}}(E)$ and $\rho_{\mathrm{V}}(E)$ from Ref. [9] used in the numerical integrations for $n$ and $h$ given by Eq. (1). The $\rho_{\mathrm{C}}(E)$ and $\rho_{\mathrm{V}}(E)$ are in tabular form and have both localized and continuum states. Their associated band edges contain the many body effects related to exchange and correlation. The independent variables are the temperature $T$ and donor density $N_{\mathrm{D}}$. The Fermi energy is varied for a given temperature until Eq. (3) is satisfied to within an error of plus or minus $10^{-3} \times N_{\mathrm{D}}$.

Numerical fitting procedures then give a closed-form analytic expression for the dependence of carrier concentration $n_{\mathrm{BGN}}$ on the Fermi energy $E_{\mathrm{F}}$; namely,

$$
\begin{aligned}
& \log _{10}\left(n_{\mathrm{BGN}} \mathrm{cm}^{3}\right) \\
& =a_{\mathrm{BGN} 0}+a_{\mathrm{BGN} 1} E_{F}+a_{\mathrm{BGN} 2} E_{F}^{2}+\ldots+a_{\mathrm{BGN} l} E_{\mathrm{F}}^{l} \ldots .
\end{aligned}
$$

During the fitting analyses, we rely substantially on graphics and keep the number of fitting parameters to a minimum, subject to the constraint that the residual standard deviation $S_{\text {res }}$ is acceptably small; i.e., $S_{\text {res }} \leq$ 0.01 . The standard deviation is a measure of the "average" error by which a fitted model represents a set of data points and thereby is a metric for assessing the quality of the fit. A smaller $S_{\text {res }}$ indicates a better fit. The residual standard deviation for a model $Y^{f}=f(Z)$ is

$$
S_{\text {res }}=\sqrt{\left[\sum_{j=1}^{N}\left(Y_{j}-\bar{Y}_{j}^{\mathrm{f}}\right)^{2} /(N-P)\right]},
$$

where $Y_{j}$ are the calculated data values, the $\bar{Y}_{j}^{\mathrm{f}}$ are the predicted values from the fitted model, $N$ is the total number of data points (here $N=28$ ), and $P$ is the total number of parameters to be fitted in the model. We use the NIST-developed DATAPLOT [15] software for both the exploratory graphics and for the statistical analyses.

In addition, we compare the above BGN model results with an equivalent two-band PDOS2 model for which there is no bandgap narrowing and for which the tabular carrier densities of states $\rho_{\mathrm{C}}(E)$ and $\rho_{\mathrm{V}}(E)$ from Ref. [9] are replaced with

$$
\begin{aligned}
& \rho_{\mathrm{C}}(E)=\frac{N_{\mathrm{C}} 4 \pi V \sqrt{E}}{\left(8 \pi^{3}\right)\left(\hbar^{2} / 2 m_{\mathrm{C}} m_{0}\right)^{3 / 2}} \text { and } \\
& \rho_{\mathrm{V}}(E)=\frac{N_{\mathrm{V}} 4 \pi V \sqrt{E}}{\left(8 \pi^{3}\right)\left(\hbar^{2} / 2 m_{\mathrm{V}} m_{0}\right)^{3 / 2}}, \text { respectively. }
\end{aligned}
$$

The corresponding polynomial fit for the carrier concentration $n_{0}$ versus Fermi energy $E_{\mathrm{F}}$ relation is then denoted by

$$
\log _{10}\left(n_{0} \mathrm{~cm}^{3}\right)=a_{00}+a_{01} E_{\mathrm{F}}+a_{02} E_{\mathrm{F}}^{2}+\ldots+a_{0 l} E_{\mathrm{F}}^{l} \ldots .
$$

Figure 1 compares the calculated carrier concentration $n_{\mathrm{BGN}}$ and $n_{0}$ data for 28 values of donor densities between $10^{16} \mathrm{~cm}^{-3}$ and $10^{19} \mathrm{~cm}^{-3}$. For a given Fermi energy, the electron densities predicted by the BGN model (solid-black curve) are typically factors of two smaller than the electron densities predicted by an equivalent parabolic two-band model (dashed-blue curve).

\subsection{PDOS Models}

We solve self-consistently, by means of an iterative procedure, Eq. (3) with Eq. (5). The independent variables are the temperature $T$ and donor density $N_{\mathrm{D}}$.

We give here the results for fitting the logarithm to the base 10 of the total electron density and the electron

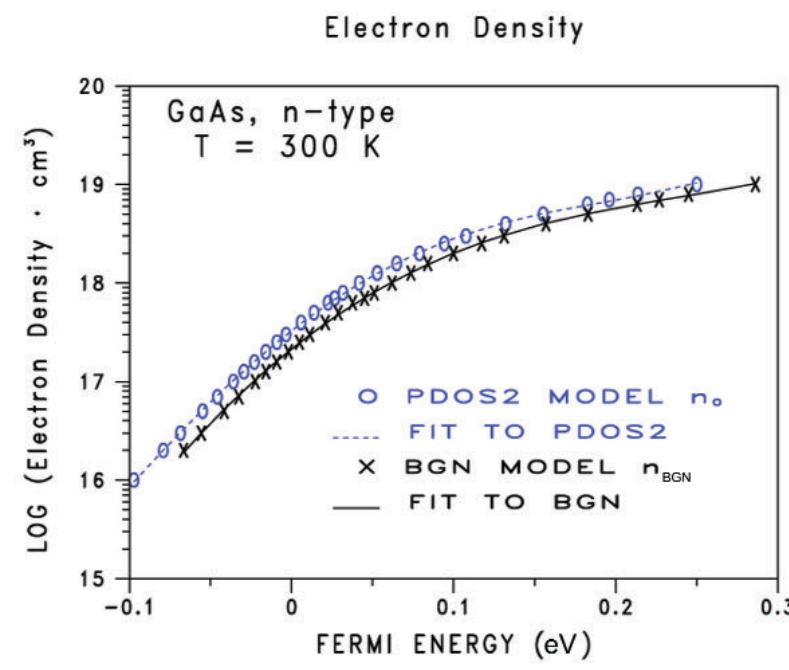

Figure 1. The calculated electron density $n_{\mathrm{BGN}}$ from the BGN model as a function of Fermi energy for $\mathrm{n}$-type GaAs at $300 \mathrm{~K}$ is given by the solid-black curve. The calculated electron density $n_{0}$ from the two-band PDOS2 model, no bandgap narrowing model, as a function of Fermi energy for n-type GaAs at $300 \mathrm{~K}$ is given by the dashedblue curve. The Fermi energy is relative to the majority conduction band edge at the $\Gamma$ symmetry point in the first Brillouin zone. 


\section{Journal of Research of the National Institute of Standards and Technology}

densities in each of the three conduction sub-bands at $\Gamma$, L, and $\mathrm{X}, n_{\Gamma}, n_{\mathrm{L}}$, and $n_{\mathrm{X}}$, respectively, to polynomials in $E_{\mathrm{F}}$, namely,

$$
\begin{aligned}
& \log _{10}\left(n_{\mathrm{t}} \mathrm{cm}^{3}\right)=a_{\mathrm{t} 0}+a_{\mathrm{t} 1} E_{\mathrm{F}}+a_{\mathrm{t} 2} E_{\mathrm{F}}^{2}+\ldots+a_{\mathrm{t} l} E_{\mathrm{F}}^{l} \ldots, \\
& \log _{10}\left(n_{\Gamma} \mathrm{cm}^{3}\right)=a_{\Gamma 0}+a_{\Gamma 1} E_{\mathrm{F}}+a_{\Gamma 2} E_{\mathrm{F}}^{2}+\ldots+a_{\Gamma l} E_{\mathrm{F}}^{l} \ldots, \\
& \log _{10}\left(n_{\mathrm{L}} \mathrm{cm}^{3}\right)=a_{\mathrm{L} 0}+a_{\mathrm{L} 1} E_{\mathrm{F}}+a_{\mathrm{L} 2} E_{\mathrm{F}}^{2}+\ldots+a_{\mathrm{L} l} E_{\mathrm{F}}^{l} \ldots, \text { and }
\end{aligned}
$$

$\log _{10}\left(n_{\mathrm{X}} \mathrm{cm}^{3}\right)=a_{\mathrm{X} 0}+a_{\mathrm{X} 1} E_{\mathrm{F}}+a_{\mathrm{X} 2} E_{\mathrm{F}}^{2}+\ldots+a_{\mathrm{X} l} E_{\mathrm{F}}^{l} \cdots$

Figures 2 and 3 give the calculated electron densities as functions of the Fermi energy. The corresponding fitted curves are not shown in Figs. 2 and 3 because the pairs of curves (calculated and fitted) for each of the electron densities $n_{\mathrm{t}}, n_{\Gamma}, n_{\mathrm{L}}$, and $n_{\mathrm{X}}$, lie on top of one another to within the line widths of each curve. Also, since the screening radii for the carriers from Eq. (2) are not needed when interpreting the proposed measurements considered here, the corresponding screening radii are not presented in this paper. Figure 2 compares the results from the PDOS2 model (dashed-blue curve with long spaces), PDOS2NPG model (solid-green curve), and PDOS4 model (dashed-red curve with short

\section{Donor Density}

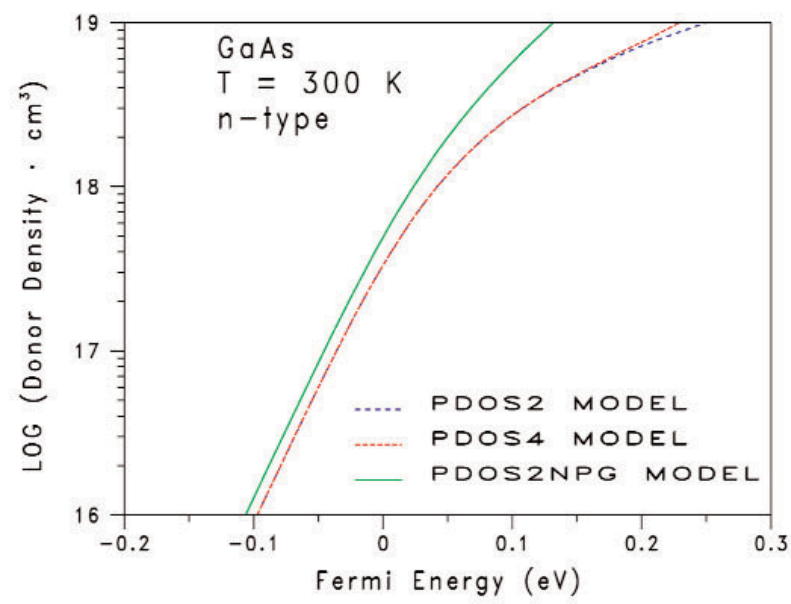

Figure 2. Comparisons among three PDOS models with and without the non-parabolic factor $\xi$ in Eq. (5) for the electron energy dispersion $E_{\mathrm{c} \Gamma}(\boldsymbol{k})$. The solid-green curve shows the results for an equivalent two-band model that has one equivalent $\Gamma$ conduction band with non-parabolicity factor $\xi$ and one equivalent $\Gamma$ valence band; that is, the PDOS2NPG model. The dashed-blue curve with long spaces shows the results for an equivalent parabolic two-band model PDOS2 $(\xi=0)$. The dashed-red curve short spaces shows the results for the four-band PDOS4 model. The Fermi energy is relative to the majority conduction band edge at the $\Gamma$ symmetry point in the first Brillouin zone.

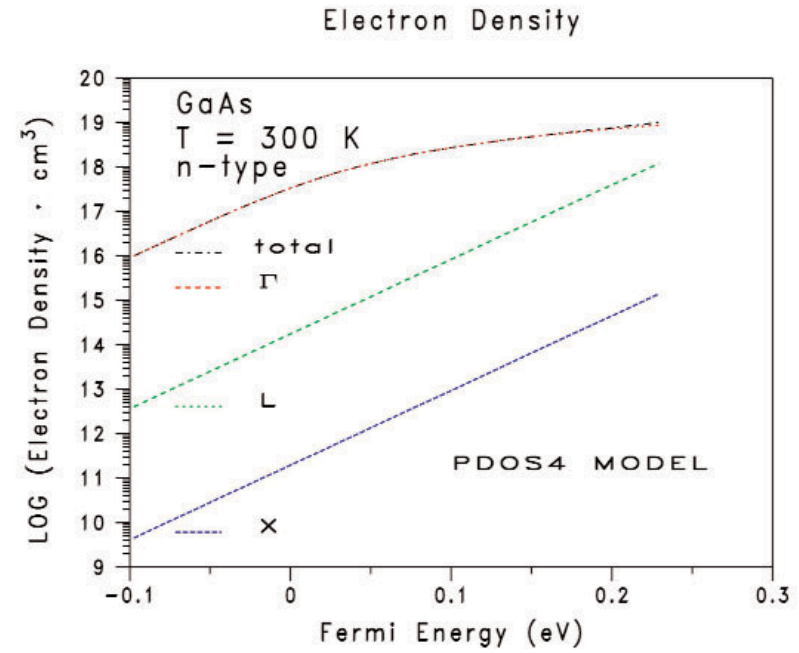

Figure 3. The calculated electron densities $n_{\mathrm{t}}, n_{\Gamma}, n_{\mathrm{L}}$, and $n_{\mathrm{X}}$ from the four-band PDOS4 model as functions of the Fermi energy for ntype GaAs at $300 \mathrm{~K}$. The Fermi energy is relative to the majority conduction band edge at the $\Gamma$ symmetry point in the first Brillouin zone.

spaces). The data for the blue curve in Fig. 1 is the same data for the blue curve in Fig. 2. Figure 3 shows the results for the four-band PDOS4 model. Unlike GaSb, most of the electrons are in the conduction sub-band at $\Gamma$. The conduction sub-band at $L$ is only weakly populated at the highest Fermi energies, and the conduction sub-band at $X$ is negligible.

\subsection{Polynomial Fits - Closed-Form Analytic Expressions}

Tables 6 to 11 give the fitting parameters for polynomial fits to $\log _{10}\left(n_{\mathrm{BGN}} \mathrm{cm}^{3}\right), \log _{10}\left(n_{0} \mathrm{~cm}^{3}\right), \log _{10}\left(n_{\mathrm{t}} \mathrm{cm}^{3}\right)$, $\log _{10}\left(n_{\Gamma} \mathrm{cm}^{3}\right), \log _{10}\left(n_{\mathrm{L}} \mathrm{cm}^{3}\right)$, and $\log _{10}\left(n_{\mathrm{X}} \mathrm{cm}^{3}\right)$ as shown, respectively, in Eqs. (21) and (24) to (28) and the corresponding residual standard deviations $S_{\text {res. }}$. In general, the values of $S_{\text {res }}$ decrease monotonically with increasing number $l$ of terms in these polynomials. But, care must be taken to avoid fitting noise in data sets. The general guideline for many data sets is that when the $t$-ratio (i.e., the absolute value of the ratio of the estimated parameter value divided by its estimated standard deviation) is less than about 2 , then the rate of decrease in $S_{\text {res }}$ with increasing $l$ tends to decrease, and proceeding with higher $l$ values probably is not warranted. Because the changes in values of $S_{\text {res }}$ between $l=4$ and $l=5$ are not experimentally significant, we use the fitting parameters for the quartic case $l=4$ in Eqs. (21) and (24). Similary, because the changes in values of $S_{\text {res }}$ between $l=3$ and $l=4$ are not experimen- 


\section{Journal of Research of the National Institute of Standards and Technology}

Table 6. Bandgap narrowing BGN model for $\log _{10}\left(n_{\mathrm{BGN}} \mathrm{cm}^{3}\right)$. The five fitting parameters for a quartic polynomial fit Eq. (21) of the theoretical calculation for the equivalent conduction band electron density in n-type, zinc blende GaAs at $300 \mathrm{~K}$ as a function of the Fermi energy relative to the bottom of the equivalent conduction band at $\Gamma$. This quartic polynomial fit, which represents the theoretical results for Eq. (3), is valid only when $-0.067 \mathrm{eV} \leq E_{\mathrm{F}} \leq 0.286 \mathrm{eV}$. The $t$-ratio is the absolute value of the estimated fitting parameter $\left|a_{\mathrm{BGN}}\right|$ divided by its estimated standard deviation. The residual standard deviation is $S_{\text {res }}=0.0130$.

\begin{tabular}{lllll}
\hline \hline Fitting parameter & Estimated value & $\begin{array}{c}\text { Estimated standard } \\
\text { deviation }\end{array}$ & Units & $t$-ratio \\
\hline$a_{\mathrm{BGN} 0}$ & 17.3292 & $0.4191 \times 10^{-2}$ & & $4.135 \times 10^{3}$ \\
$a_{\mathrm{BGN} 1}$ & 13.1545 & $0.7646 \times 10^{-1}$ & $\mathrm{eV}^{-1}$ & $1.72 \times 10^{2}$ \\
$a_{\mathrm{BGN} 2}$ & -37.4789 & 1.473 & $\mathrm{eV}^{-2}$ & 25.45 \\
$a_{\mathrm{BGN} 3}$ & 26.5678 & 12.86 & $\mathrm{eV}^{-3}$ & 2.067 \\
$a_{\mathrm{BGN} 4}$ & 53.7760 & 29.43 & $\mathrm{eV}^{-4}$ & 1.827 \\
\hline
\end{tabular}

Table 7. Two-band, no bandgap narrowing PDOS2 model for $\log _{10}\left(n_{0} \mathrm{~cm}^{3}\right)$. The five fitting parameters for a quartic polynomial fit Eq. (24) of the theoretical calculation for the L sub-band electron density in n-type, zinc blende GaAs at $300 \mathrm{~K}$ as a function of the Fermi energy relative to the bottom of the conduction $\Gamma$ sub-band. This quartic polynomial fit, which represents the theoretical results for Eq. (3), is valid only when $-0.0974 \mathrm{eV} \leq E_{\mathrm{F}} \leq 0.250 \mathrm{eV}$. The $t$-ratio is the absolute value of the estimated fitting parameter $\left|a_{0 i}\right|$ divided by the its estimated standard deviation. The residual standard deviation is $S_{\text {res }}=0.0122$.

\begin{tabular}{lllll}
\hline \hline Fitting parameter & Estimated value & $\begin{array}{c}\text { Estimated standard } \\
\text { deviation }\end{array}$ & Units & $t$-ratio \\
\hline$a_{00}$ & 17.5156 & $0.3411 \times 10^{-2}$ & & $5.136 \times 10^{3}$ \\
$a_{01}$ & 12.8520 & $0.8063 \times 10^{-1}$ & $\mathrm{eV}^{-1}$ & $3.1 \times 10^{2}$ \\
$a_{02}$ & -34.5783 & 0.6672 & $\mathrm{eV}^{-2}$ & 51.83 \\
$a_{03}$ & -27.3728 & 9.166 & $\mathrm{eV}^{-3}$ & 2.986 \\
$a_{04}$ & 223.720 & 29.54 & $\mathrm{eV}^{-4}$ & 7.573 \\
\hline
\end{tabular}

Table 8. Four-band PDOS4 model for total electron density $\log _{10}\left(n_{\mathrm{t}} \mathrm{cm}^{3}\right)$. The four fitting parameters for a cubic polynomial fit Eq. (25) of the theoretical calculation for the total electron density in n-type, zinc blende GaAs at $300 \mathrm{~K}$ as a function of the Fermi energy relative to the bottom of the conduction $\Gamma$ sub-band. This cubic polynomial fit, which represents the theoretical results for Eq. (3), is valid only when $-0.0974 \mathrm{eV} \leq E_{\mathrm{F}} \leq 0.229 \mathrm{eV}$. The $t$-ratio is the absolute value of the estimated fitting parameter $\left|a_{\mathrm{t} i}\right|$ divided by its estimated standard deviation. The residual standard deviation is $S_{\text {res }}=0.0234$.

\begin{tabular}{lllll}
\hline \hline Fitting parameter & Estimated value & $\begin{array}{c}\text { Estimated standard } \\
\text { deviation }\end{array}$ & Units & $t$-ratio \\
\hline$a_{\mathrm{t} 0}$ & 17.5198 & $0.6519 \times 10^{-2}$ & & $2.687 \times 10^{3}$ \\
$a_{\mathrm{t} 1}$ & 12.3461 & $0.9401 \times 10^{-1}$ & $\mathrm{eV}^{-1}$ & $1.31 \times 10^{2}$ \\
$a_{\mathrm{t} 2}$ & -35.5542 & 1.322 & $\mathrm{eV}^{-2}$ & 26.90 \\
$a_{\mathrm{t} 3}$ & 40.1630 & 6.488 & $\mathrm{eV}^{-3}$ & 6.190 \\
\hline
\end{tabular}


Table 9. Four-band PDOS4 model for electron density in the $\Gamma$ sub-band $\log _{10}\left(n_{\Gamma} \mathrm{cm}^{3}\right)$. The four fitting parameters for a cubic polynomial fit Eq. (26) of the theoretical calculation for the $\Gamma$ sub-band electron density in n-type, zinc blende GaAs at $300 \mathrm{~K}$ as a function of the Fermi energy relative to the bottom of the conduction $\Gamma$ sub-band. This cubic polynomial fit, which represents the theoretical results for Eq. (3), is valid only when $-0.0974 \mathrm{eV} \leq E_{\mathrm{F}} \leq 0.229 \mathrm{eV}$. The $t$-ratio is the absolute value of the estimated fitting parameter $\left|a_{\Gamma i}\right|$ divided by its estimated standard deviation. The residual standard deviation is $S_{\text {res }}=0.0214$.

\begin{tabular}{lllll}
\hline \hline Fitting parameter & Estimated value & $\begin{array}{c}\text { Estimated standard } \\
\text { deviation }\end{array}$ & Units & $t$-ratio \\
\hline$a_{\Gamma 0}$ & 17.5187 & $0.5978 \times 10^{-2}$ & & $2.930 \times 10^{3}$ \\
$a_{\Gamma 1}$ & 12.3944 & $0.8621 \times 10^{-1}$ & $\mathrm{eV}^{-1}$ & $1.438 \times 10^{2}$ \\
$a_{\Gamma 2}$ & -35.1795 & 1.212 & $\mathrm{eV}^{-2}$ & 29.03 \\
$a_{\Gamma 3}$ & 33.7647 & 5.950 & $\mathrm{eV}^{-3}$ & 5.675 \\
\hline
\end{tabular}

Table 10. Four-band PDOS4 model for electron density in the L sub-band $\log _{10}\left(n_{\mathrm{L}} \mathrm{cm}^{3}\right)$. The four fitting parameters for a cubic polynomial fit Eq. (27) of the theoretical calculation for the L sub-band electron density in n-type, zinc blende GaAs at $300 \mathrm{~K}$ as a function of the Fermi energy relative to the bottom of the conduction $\Gamma$ sub-band. This cubic polynomial fit, which represents the theoretical results for Eq. (3), is valid only when $-0.0974 \mathrm{eV} \leq E_{\mathrm{F}} \leq 0.229 \mathrm{eV}$. The $t$-ratio is the absolute value of the estimated fitting parameter $\left|a_{\mathrm{L} i}\right|$ divided by its estimated standard deviation. The residual standard deviation is $S_{\text {res }}=0.00117$.

\begin{tabular}{lllll}
\hline \hline Fitting parameter & Estimated value & $\begin{array}{c}\text { Estimated standard } \\
\text { deviation }\end{array}$ & Units & $t$-ratio \\
\hline$a_{\mathrm{L} 0}$ & 14.2422 & $0.3269 \times 10^{-3}$ & & $4.357 \times 10^{4}$ \\
$a_{\mathrm{L} 1}$ & 16.8180 & $0.4714 \times 10^{-2}$ & $\mathrm{eV}^{-1}$ & $3.568 \times 10^{3}$ \\
$a_{\mathrm{L} 2}$ & 0.162418 & $0.6627 \times 10^{-1}$ & $\mathrm{eV}^{-2}$ & 2.451 \\
$a_{\mathrm{L} 3}$ & -2.1988 & 0.3253 & $\mathrm{eV}^{-3}$ & 6.759 \\
\hline
\end{tabular}

Table 11. Four-band PDOS4 model for the electron density X sub-band $\log _{10}\left(n_{\mathrm{X}} \mathrm{cm}^{3}\right)$. The four fitting parameters for a cubic polynomial fit Eq. (28) of the theoretical calculation for the X sub-band electron density in n-type, zinc blende GaAs at $300 \mathrm{~K}$ as a function of the Fermi energy relative to the bottom of the conduction $\Gamma$ sub-band. This cubic polynomial fit, which represents the theoretical results for Eq. (3), is valid only when $-0.0974 \mathrm{eV} \leq E_{\mathrm{F}} \leq 0.229 \mathrm{eV}$. The $t$-ratio is the absolute value of the estimated fitting parameter $\left|a_{\mathrm{X} i}\right|$ divided by its estimated standard deviation. The residual standard deviation is $S_{\text {res }}=0.0000195$.

\begin{tabular}{lllll}
\hline \hline Fitting parameter & Estimated value & $\begin{array}{c}\text { Estimated standard } \\
\text { deviation }\end{array}$ & Units & t-ratio \\
\hline$a_{\mathrm{X} 0}$ & 11.2919 & $0.5452 \times 10^{-5}$ & & $2.071 \times 10^{6}$ \\
$a_{\mathrm{X} 1}$ & 16.7999 & $0.7862 \times 10^{-4}$ & $\mathrm{eV}^{-1}$ & $2.137 \times 10^{5}$ \\
$a_{\mathrm{X} 2}$ & $-0.54216 \times 10^{-3}$ & $0.1105 \times 10^{-2}$ & $\mathrm{eV}^{-2}$ & 0.4905 \\
$a_{\mathrm{X} 3}$ & $-0.4041 \times 10^{-2}$ & $0.5426 \times 10^{-2}$ & $\mathrm{eV}^{-3}$ & 0.7448 \\
\hline
\end{tabular}


tally significant, we use the fitting parameters for the cubic $l=3$ case in Eqs. (25) to (28).

\section{Conclusions}

The foregoing theory for extracting electron densities from Raman spectra is unique in two ways: 1) It treats the many-body effects self-consistently, and 2) it is valid at room temperature for arbitrary values of the ratio $R=\left(Q^{2} / \alpha\right)$. When high concentrations of carriers exists, this theory and its associated numerical procedures for determining carrier concentrations from Fermi energies are necessary for interpreting room temperature Raman spectra self-consistently for arbitrary values of the ratio $R$, even for $R \approx 1$. The BGN models presented here include many-body quantum effects and bandgap narrowing. However, obtaining Fermi energies from experimental Raman spectra is beyond the scope of the present paper and involves the following steps to implement contactless measurements of carrier densities:

1) Numerical evaluation of Eq. (17) with adaptive grids to treat the integrable singularities of the integrands,

2) Three-dimensional visualization of computer results for predicted Raman line shapes as functions of Fermi energy and frequency, and

3) Iteration of the predicted Raman line shape with the Fermi energy as a variation parameter to give the best self-consistent fit to the measured Raman line shape.

\section{Acknowledgments}

The author acknowledges many discussions with James Maslar concerning physical models for interpreting Raman measurements. He thanks Alan Heckert for guidance in using DATAPLOT and James Filliben for help in obtaining the closed-form analytic expressions for carrier densities from numerical tabulations of theoretical calculations. He benefited substantially from having access to computers in the NIST Information Technology Laboratory (ITL) and from support given by other ITL staff members, particularly, Howard Hung, Judith Terrill, and James Sims.

\section{References}

[1] 2003 International Technology Roadmap for Semiconductors, International SEMATECH, Austin, Texas, http://www.itrs.net/ Links/2003ITRS/Home2003.htm.
[2] 2004 iNEMI Technology Roadmaps, International Electronics Manufacturing Initiative, Inc., Herndon, VA, http://www.inemi. org/cms/roadmapping/2004_iNEMI_Roadmap.html.

[3] Chemical Industry R\&D Roadmap for Nanomaterials by Design: From Fundamentals to Function, December 2003, Chemical Industry Vision 2020 Technology Partnership, Baltimore, MD, http://www.chemicalvision2020.org/nanoma terialsroadmap.html.

[4] S. Ernst, A. R. Goñi, K. Syassen, and M. Cardona, Plasmon Raman Scattering and Photoluminescence of Heavily Doped ntype InP Near the $\Gamma$-X Crossover, Phys. Rev. B53, 1287 (1996). See Eq. (A4), p. 1291, therein.

[5] L. Artús, R. Cuscó, J. Ibáñez, N. Blanco, and G. González-Días, Raman Scattering by LO Phonon-Plasmon Coupled Modes in n-type InP, Phys. Rev. B60, 5456 (1999). See Eq. (5.4), p. 5462, therein.

[6] W. Richter, U. Nowak, H. Jürgensen, and U. Rössler, Electron Plasma in a Non-Parabolic Conduction Band, Solid State Communications 67, 199 (1988). See Eq. (10), p. 201, therein.

[7] R. Cuscó, L. Artús, S. Hernández, J. Ibáñez, and M. Hopkinson, Raman Scattering by LO Phonon-Plasmon Coupled Modes in n-type $\mathrm{In}_{0.53} \mathrm{Ga}_{0.47}$ As, Phys. Rev. B65, 035210-1 (2001).

[8] G. Abstreiter, M. Cardona, and A. Pinczuk, in Light Scattering in Solids IV, Chapter 2 Light Scattering by Free Carrier Excitations in Semiconductors, edited by M. Cardona and G. Güntherodt, Springer, New York (1984) pp. 5-150, Eq. (2.108), p. 55 and references therein.

[9] H. S. Bennett, High Dopant and Carrier Concentration Effects in Gallium Aluminum Arsenide: Densities of States and Effective Intrinsic Carrier Concentrations, J. Appl. Phys. 83, 3102 (1998).

[10] H. S. Bennett and Howard Hung, Dependence of Electron Density on Fermi Energy in N-Type Gallium Arsenide, J. Res. Natl. Inst. Stand. Technol. 108, 193 (2003).

[11] J. S. Blakemore, Semiconducting and Other Major Properties of Gallium Arsenide, J. Appl. Phys. 53, R123 (1982).

[12] M. E. Levinshtein and S. Rumyantsev, in Handbook Series on Semiconductor Parameters, Volume I, edited by $\mathrm{M}$. Levinshtein, S. Rumyantsev, and M. Shur, World Scientific Publishing, Singapore (1996) Capter 4, pp. 77-103.

[13] T. Lindhard, On The Properties of a Gas of Charged Particles, Dan. Mat. Fys. Medd. 28 8, 1 (1954).

[14] N. D. Mermin, Lindhard Dielectric Function in the RelaxationTime Approximation, Phys. Rev. B1, 2362 (1970).

[15] J. J. Filliben and A. N. Heckert, the DATAPLOT software for graphics and detailed statistical analyses runs on both UNIX and WINTEL platforms. It has both command-line versions and graphical user interface (GUI) versions. It is available by downloading from http://www.itl.nist.gov/div898/software/dataplot/. In addition, the NIST-SEMATECH Engineering Statistics Handbook at http://www.itl.nist.gov/div898/handbook/ is based in part on DATAPLOT. This latter website has tutorials that explain in some detail the statistical analysis methods used in this paper.

About the authors: Herbert S. Bennett is a NIST Fellow in the Semiconductor Electronics Division of the Electronics and Electrical Engineering Laboratory. He is a Life Fellow of the Institute of Electrical and Electronics Engineers and a Fellow of the American Physical Society. His research interests include 
Volume 112, Number 4, July-August 2007

Journal of Research of the National Institute of Standards and Technology

improved understanding, metrology, and standards for nanoelectronics, optoelectronics, and magnetic materials and assessing the quality of digital images such as those used in medical imaging. He is active with international industrial consensus building for technology roadmaps on semiconductors and with international standards development for nanoelectrotechnology. The National Institute of Standards and Technology is an agency of the U.S. Department of Commerce. 